\title{
HISTÓRIA DA NEUROCIRURGIA NO RIO DE JANEIRO
}

\author{
Sebastião Silva Gusmão ${ }^{1}$
}

RESUMO - Relata-se a vida profissional dos precursores e pioneiros que possibilitaram o nascimento da neurocirurgia no Rio de Janeiro.

PALAVRAS-CHAVE: neurocirurgia, história.

History of neurosurgery in Rio de Janeiro

ABSTRACT - The professional life of the pioneers of neurological surgery in Rio de Janeiro is presented.

KEY WORDS: neurosurgery, history.

As conquistas essenciais para o desenvolvimento da neurocirurgia moderna foram o avanço da cirurgia geral, especialmente a anestesia (Morton, 1846) e a antissepsia (Lister, 1867) e a teoria das localizações cerebrais (Broca, 1861) ${ }^{1,2}$. Ela foi estabelecida nas duas últimas décadas do século XIX e primeiras décadas do século XX graças, principalmente, aos pioneiros Victor Horsley (1857-1916) e Harvey Cushing (1864-1939) ${ }^{1-3}$.

No Brasil, o nascimento da neurocirurgia dependeu também do desenvolvimento prévio da cirurgia e da anestesia. O ensino oficial da neurologia foi inaugurado no Brasil em 1912, quando da criação da Disciplina de Neurologia, distinta da Psiquiatria, na Faculdade de Medicina do Rio de Janeiro, sendo designado para regê-la Antônio Austregésilo Rodrigues Lima (1876 - 1961), que chefiava o Serviço de Neurologia da Santa Casa de Misericórdia do Rio de Janeiro ${ }^{4}$. A cirurgia moderna no Brasil teve início no final do século XIX, especialmente no Rio de Janeiro, com as obras de Cândido Borges Monteiro, Chapot-Prévost, Andrade Pertence, Domingos de Góes eVasconcelos, Paes Lemee Augusto Brandão Filho ${ }^{5}$. Este último era denominado o Príncipe da Cirurgia Brasileira e pode ser considerado o precursor da neurocirurgia brasileira, pois foi o primeiro cirurgião geral a ir além da cirurgia craniana do trauma, tentando a cirurgia dos tumores cerebrais e da neuralgia do trigêmeo e iniciando, em nosso meio, os exames neurorradiológicos (ventriculografia eangiografia cerebral).

No final da terceira década do século XX, a mo- derna cirurgia e a neurologia estavam bem assentadas em nosso meio, especialmente no Rio de Janeiro, propiciando as condições para o nascimento da neurocirurgia brasileira. O ano de 1928 pode ser considerado como a data crucial da neurocirurgia brasileira. Nesteano, Brandão Filho encontrava-se no auge de sua tentativa de tratamento cirúrgico dos tumores cerebrais e, enquanto realizava, sob a orientação de Egas Moniz (1874 - 1955), a primeira angiografia cerebral no país, Antônio Austregésilo encontrava-se visitando os serviços de neurocirurgia dos Estados Unidos. De regresso, convoca Alfredo Monteiro ej osé Ribe Portugal para o início da neurocirurgia brasileira como especialidade ${ }^{6}$.

\section{AUGUSTO BRANDÃO FILHO}

Augusto Brandão Filho (1881 - 1957) (Fig 1) foi professor de Clínica Cirúrgica da Faculdade Nacional de Medicina da Universidade do Brasil. Exerceu sua atividade cirúrgica no Hospital da Misericórdia, no Rio de Janeiro. Foi um dos mais hábeis cirurgiões de seu tempo e tinha também fino espírito científico. Foi o primeiro brasileiro a ir além da cirurgia do trauma e tentar o tratamento cirúrgico dos tumores cerebrais. Foi também o pioneiro dos exames neuroradiológicos em nosso país. Foi o primeiro a realizar no Brasil a ventriculografia ea angiografia cerebral. Na realização destes exames contou com a colaboração de dois grandes vultos da medicina. Na ventriculografia foi ajudado por Manoel de Abreu (1894 1962), futuro inventor, em 1936, da fotografia da imagem fluoroscópica, conhecida como abreugra-

\footnotetext{
${ }^{1}$ Faculdade de Medicina da Universidade Federal de Minas Gerais (UFMG), Belo Horizonte MG, Brasil.
} 


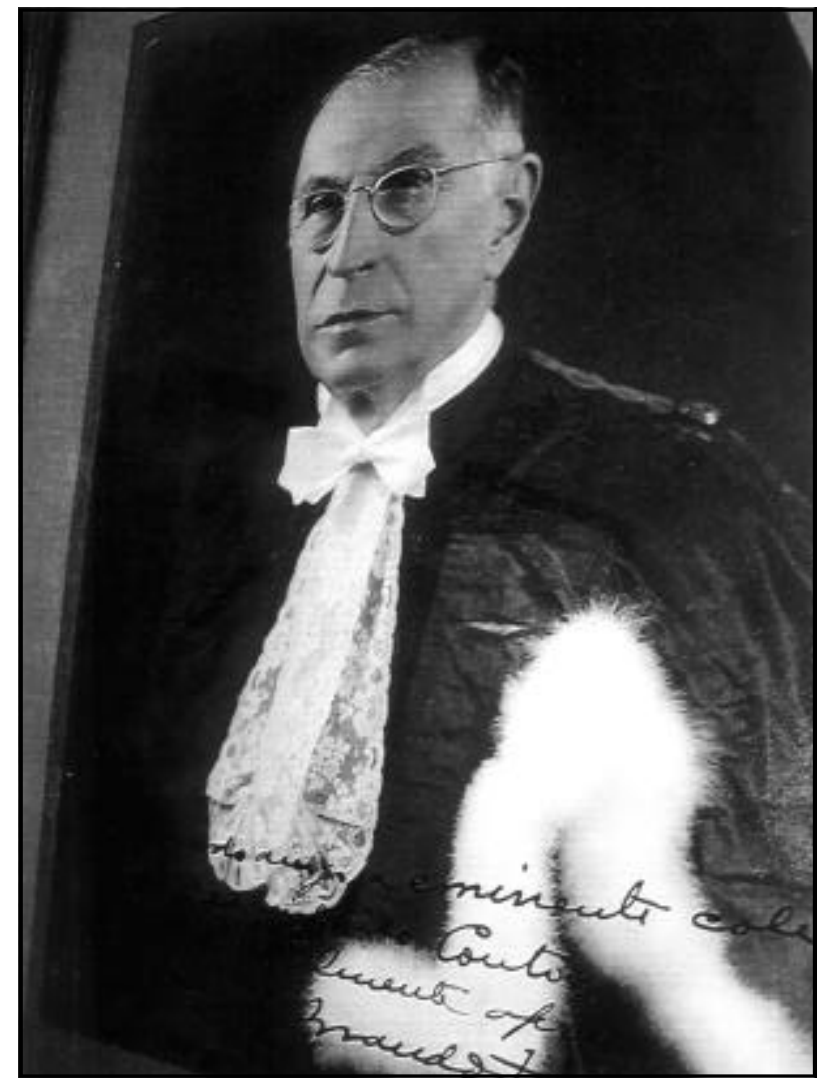

Fig 1. Brandão Filho (1881 - 1957).

$\mathrm{fia}^{7}$. Na angiografia cerebral foi auxiliado pelo próprio inventor do método, Egas Moniz ${ }^{8,9}$, que em 1928, encontrava-se em visita ao Brasil (Figs 2,3).

No livro Tumores do encéfalo: algumas observações comentadas ${ }^{10}$ relata seis casos de intervenção sobre o crânio operados no período de 1927 a 1931 ea estes acrescenta um sétimo publicado em 19247. Todos os casos foram operados em fase avançada de hipertensão intracraniana e faleceram. A indica-

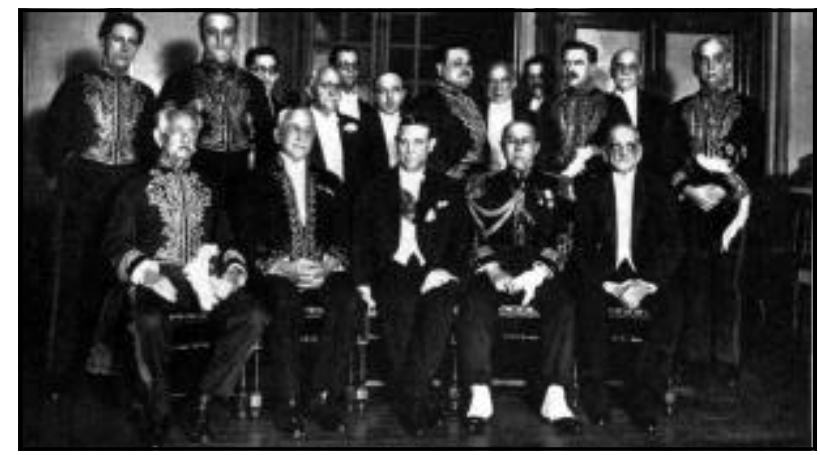

Fig 2. Egas Moniz na Academia Brasileira de Letras. "Recepção solene na Academia Brasileira de Letras, em 22 de agosto de 1928. Entre a assistência: Dr. Medeiros e Albuquerque, Prof. Miguel Couto, Prof. Fernando de Magalhães, Dr. Ataulfo de Paiva, Dr. Humberto de Campos, Dr. Gustavo Barroso, Dr. Olegário Mariano"8. ção cirúrgica baseava-se apenas no exame neurológico e no exame radiológico simples do crânio. Apenas um caso foi submetido à ventriculografia.

Brandão Filho faz comentários pormenorizados sobre as causas dos erros de localização e sobre o insucesso do tratamento, tendo como base os grandes mestres da neurocirurgia do começo do século XX. Em cinco dos casos expôs o quiasma óptico e em dois, a fossa posterior. Em dois casos de exposição, do quiasma óptico não foi encontrado o tumor, o qual estava situado em outra região, como ficou demonstrado pela necropsia. A exploração da região do quiasma baseava-se no déficit visual e no exame radiológico simples de crânio que mostrava deformação da sela turca. Brandão Filho, com base nos trabalhos da literatura, identifica a causa do erro como sendo devida à não diferenciação das alterações da sela turca por acometimento primário de tumor hipofisário e por hipertensão intracraniana. Um tumor da fossa posterior, possivelmenteneurinoma do acústico, não foi identificado devido à não exposição do ângulo ponto-cerebelar. O sétimo caso, submetido à ventriculografia, tratava-se de hidrocefalia e foi submetido à craniectomia da fossa posterior. Além da cirurgia dos tumores cerebrais, Brandão Filho realizou também o tratamento cirúrgico da neuralgia do trigêmeo. Relata dois casos, um operado em 1922 e outro em 1923 por meio da secção da raiz sensitiva do trigêmeo ${ }^{11}$.

Brandão Filho expunha em pormenor a técnica cirúrgica empregada por meio de texto eilustrações precisos. Em seus comentários demonstrava estar a par dos trabalhos dos grandes mestres da neurocirurgia do começo do século XX. Além do título de «príncipe dos cirurgiões», merece também o de precursor da neurocirurgia no Brasil.

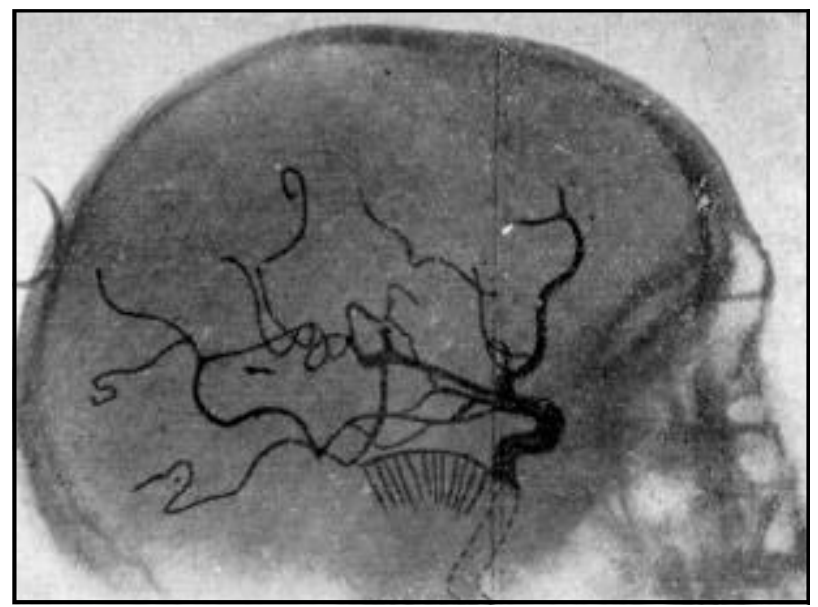

Fig 3. Imagem em perfil da primeira angiografia cerebral realizada no Brasil por Brandão Filho e Egas Moniz, em 6 de agosto de 1928. 


\section{ALFREDO ALBERTO PEREIRA MONTEIRO}

Alfredo Alberto Pereira Monteiro (1891-1961) graduou-se pela Faculdade de Medicina do Rio de Janeiro em 1914, obtendo no mesmo ano, com apenas 23 anos, a Livre-docência de anatomia da referida faculdade. Em 1928, Antônio Austregésilo convoca-o a iniciar a neurocirurgia no Brasil. No mesmo ano, juntamente com seu assistente José Ribe Portugal, inicia esta especialidade, realizando os procedimentos cirúrgicos na Santa Casa do Rio de Janeiro. Em 1932, é investido na recém criada Cátedra de Neurocirurgia da Faculdade Nacional de Medicina do Rio de Janeiro. Em 1935, abandona a especialidade e se transfere para a Cátedra de Técnica Operatória e de Cirurgia Experimental. Apesar de abandonar a especialidade, Alfredo Monteiro foi o primeiro professor de neurocirurgia no Brasil e deu impulso à especialidade, publicando vários trabalhos sobre o assunto. No seu tratado de Técnica Cirúrgica, em três volumes, deu importância destacada às técnicas neurocirúrgicas. Merece, juntamente com Brandão Filho, o título de precursor da neurocirurgia brasileira6.

\section{JOSÉ RIBE PORTUGAL}

Antônio Austregésilo (1876 - 1960), além de pioneiro da neurologia brasileira, indicou o caminho da

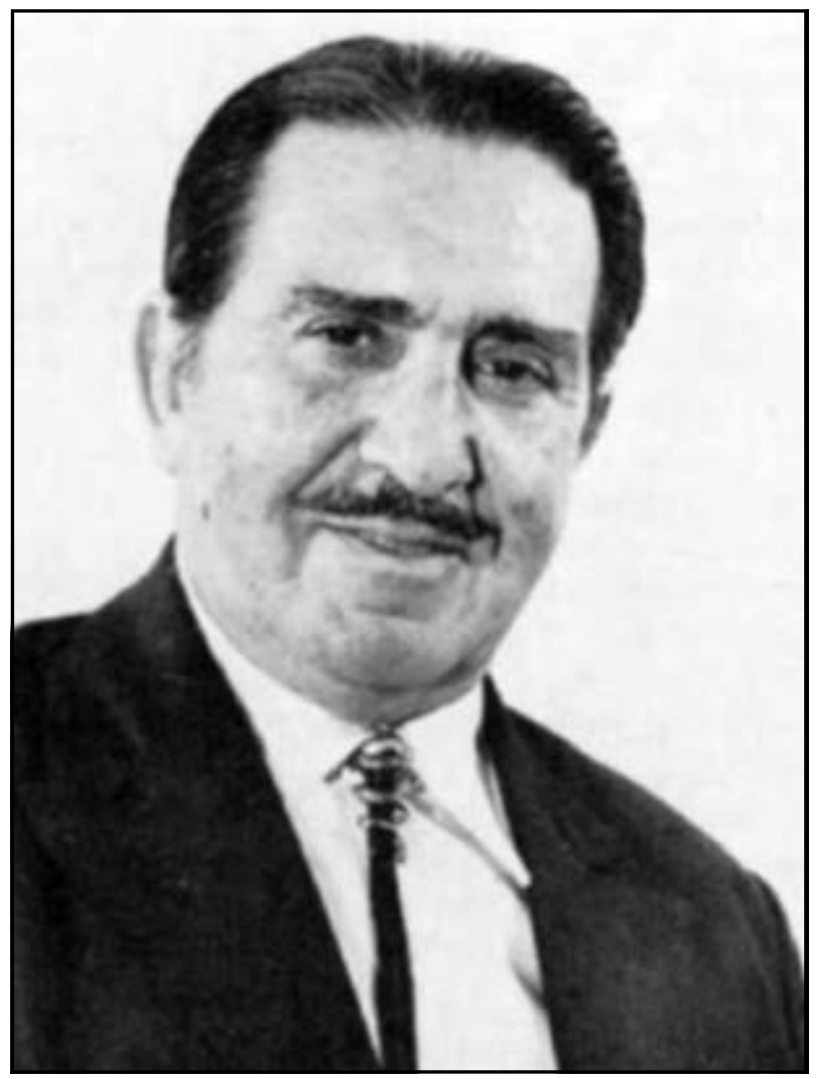

Fig 4. José Ribe Portugal (1901 - 1992). neurocirurgia a J osé Ribe Portugal. Em 1928, visita nos Estados Unidos os serviços de Cushing e Frazier. Ficou vivamente impressionado com a neurocirurgia americana e com os métodos precisos de diagnóstico, que naquela época eram a ventriculografia e a pneumoencefalografia, idealizadas por Dandy em 1918. Imediatamente após seu regresso, faz criar o Serviço de Neurocirurgia. Convoca Alfredo Alberto Pereira Monteiro, brilhante cirurgião-geral e catedrático de anatomia, o qual escolheu para seu assistente José Ribe Portugal que participava como assistente de sua disciplina de Técnica Operatória e Cirurgia Experimental ${ }^{12,13}$.

José Ribe Portugal (1901 - 1992) (Fig 4) graduouse pela Faculdade de Medicina da Universidade do Rio de Janeiro em 1927 e, no ano seguinte, como prêmio à sua proficiência, é nomeado Professor Assistente da Cadeira de Anatomia desta mesma Faculdade. Em 1928, inicia com Alfredo Monteiro os primeiros procedimentos cirúrgicos na Santa Casa de Misericórdia do Rio de Janeiro. Quando Alfredo Monteiro abandona o encargo, Portugal decide dedicar-se completamente à neurocirurgia. Em 1929, Portugal, aos 28 anos de idade, submete-se aos exames para Livre Docência de Técnica Operatória e Cirurgia Experimental na Faculdade de Medicina do Rio de Janeiro, com tese intitulada Contribuição àneurotomia retrogasseriana. O tratamento da neuralgia do trigêmeo foi seu alvo de preocupação durante toda a sua atividade como neurocirurgião (Fig 5). Em 1930, Portugal foi nomeado assistente extranumerário da Cadeira de Medicina Operatória da Faculdade de Medicina do Rio de Janeiro. Em 1932, assume a chefia do recém-criado Serviço de Neurocirurgia do Hospital da Ordem Terceira do Carmo, com cem leitos.

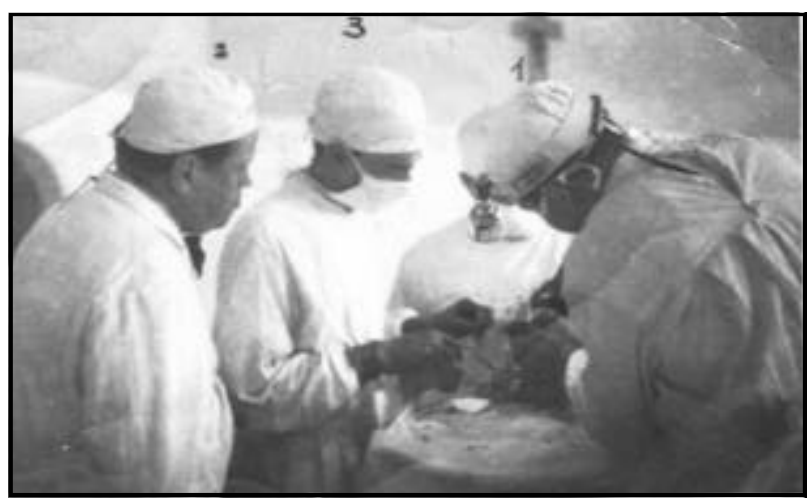

Fig 5. Neurotomia retrogasseriana diferenciada realizada em agosto de 1938, na Clínica Neurológica da Universidade do Brasil, Rio de Janeiro. Operação efetuada por José Ribe Portugal (1), assistida por Leriche (2) e auxiliada por Renato Tavares Barbosa. (3). 


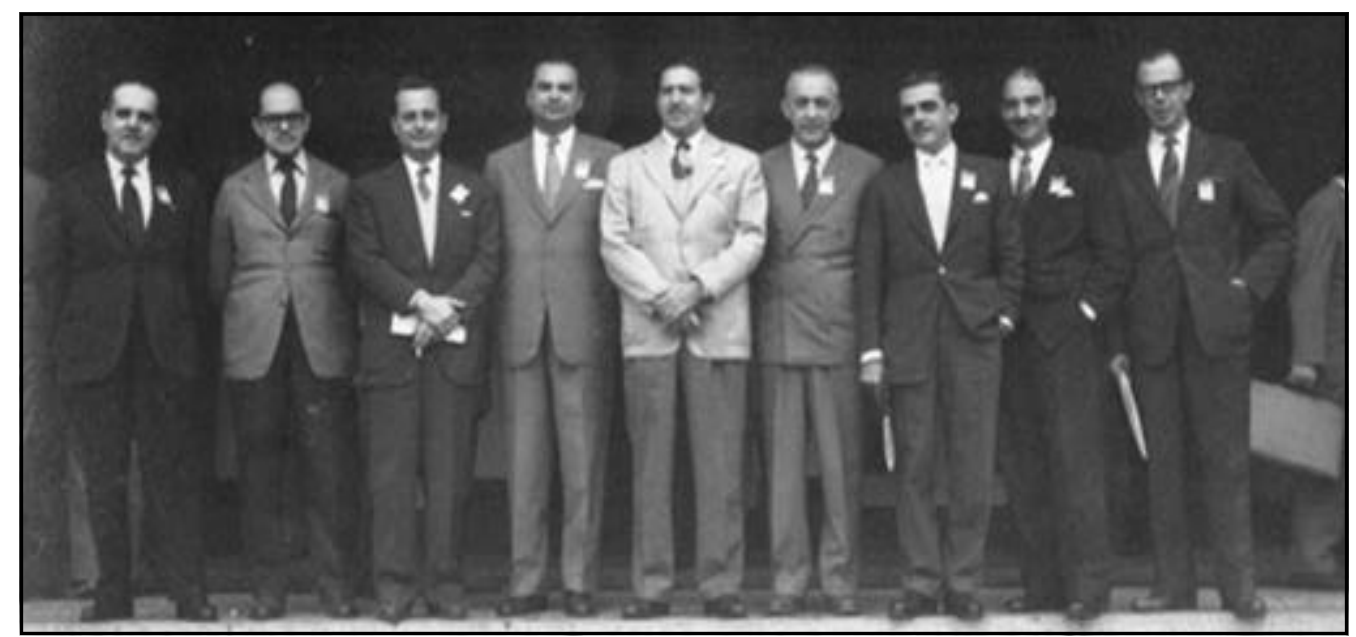

Fig 6. Primeiro Congresso da Sociedade Brasileira de Neurocirurgia realizado em Petrópolis, em 1958. Da esquerda para a direita: Lélio Gomes, Paulo Niemeyer, Rolando Tenuto, José Geraldo Albernaz, José Ribe Portugal, Mattos Pimenta, Manoel Caetano de Barros, Renato Tavares Barbosa e Francisco Rocha.

No início, Portugal foi um autodidata. Sua prática era guiada pela literatura neurocirúrgica e pela correspondência com os grandes mestres da neurocirurgia da época: Cushing, Frazier e Adson. Persistiu no aprimoramento técnico, cercando-se deaparelhagem e instalações modernas, educou um corpo de auxiliares, ministrando cursose proferindo conferências. Em 1930, Portugal passou a freqüentar o Serviço de Manuel Balado (1897 - 1942), em Buenos Aires, em rápidas visitas anuais. Em 1945, visita os serviços de John Scarff, Ingraham, Matson, Grant, Gross e Dandy. Após o Congresso Mundial de Neurocirurgia em Paris, visita os serviços de Olivecrona, Sjoqvist, Norman Dott e Jefferson. Prosseguindo em sua brilhante carreira, Portugal passou a ocupar, mais tarde, a Cátedra de Neurocirurgia na Faculdade de Ciências Médicas do Rio de Janeiro. Em 1965, passou a atuar no serviço do Hospital de Clínicas, do qual se aposentou em 1970. Em Bruxelas, Bélgica, durante o Primeiro Congresso Internacional de Cirurgia Neurológica, por iniciativa de José Ribe Portugal e José Geraldo Albernaz, foi fundada, em 26 de julho de 1957, a Sociedade Brasileira de Neurocirurgia. O Primeiro Congresso da Sociedade Brasileira de Neurocirurgia foi realizado em Petrópolis, no Hotel Quitandinha de 18 a 20 de julho de 1958 (Fig 6).

Portugal éautor de várias publicações neurocirúrgicas, distinguindo-se as que se referem à neuralgia do trigêmeo e aos meningiomas. Foi cirurgião exímio, possuidor de técnica primorosa ${ }^{14,6}$.

Como mestre de didática insuperável, formou uma plêiade de discípulos que se transformaram em grandes mestres: Renato Tavares Barbosa, Pedro
Sampaio, Otoide Pinheiro, Feliciano Pinto, Gianni Maurélio Temponi e Mário Brock.

Renato Tavares Barbosa treinou com Leksell, na Suécia, e iniciou a cirurgia estereotáxica no Brasil. Foi um dos fundadores da Sociedade Brasileira de Neurocirurgia, sendo seu presidente no biênio 1971 - 1972. Dirigiu o Serviço de Neurocirurgia do Hospital Lagoa do Rio de Janeiro, durante 27 anos.

Pedro Sampaio foi titular da Cátedra de Neurocirurgia da Faculdade de Medicina do Rio de Janeiro. Organizou o Serviço de Neurocirurgia do Hospital Pedro Ernesto, onde formou vários discípulos.

Feliciano Pinto dirigiu-se, em 1952, para Colônia, onde complementa sua formação neurocirúrgica com Wilhelm Tonnis. Em 1953, cria o Serviço de Neurologia do Instituto Nacional de Câncer do Ministério da Saúde e, em 1959, o Serviço de Neurocirurgia do Hospital Adventista Silvestre no Rio de Janeiro, onde formou vários neurocirurgiões. Foi presidente da Academia Brasileira de Neurocirurgia em 1982 e é secretário geral desta Academia desde 1979.

Gianni Maurélio Temponi, a partir de 1972, torna-se Professor Titular de Neurocirurgia da Universidade Federal do Rio de Janeiro e chefia o Serviço de Neurocirurgia do Instituto de Neurologia desta mesma Universidade.

Otoíde Pinheiro é Professor Adjunto e Livre Docenteem Anatomia e Neurocirurgia da Escola de Medicina e Cirurgia do Rio de Janeiro. A partir de 1976 assumiu a chefia do Serviço de Neurocirurgia do Hospital da Venerável Ordem Terceira de São Francisco da Penitência. Foi Presidente da Academia Brasileira de Neurocirurgia no biênio 1992-1993. 


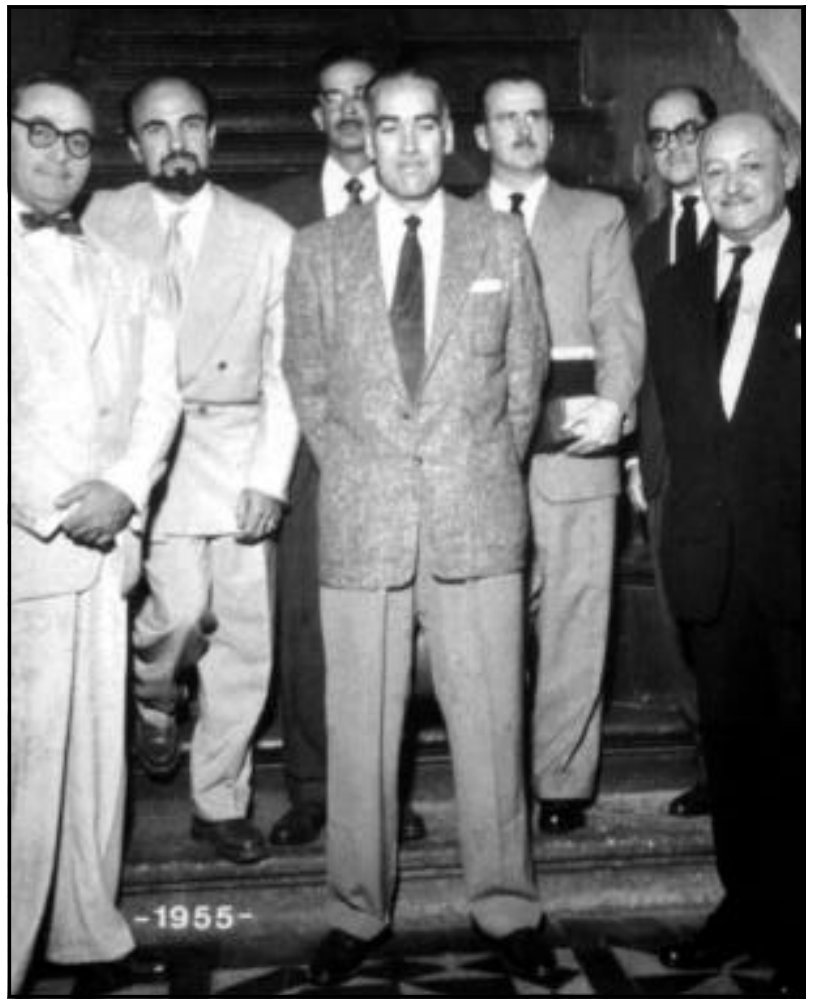

Fig 7. Colóquio Internacional de Eletrocorticografia realizado no Rio de Janeiro, em 1955, vendo-se da esquerda para a direita: Abraham Mosovich (Argentina), Henri Gastaut (França), Aristides Leão (Brasil) Earl Walker (Estados Unidos), Bartolomé Fuster (Uruguai), Paulo Niemeyer (Brasil) e Carlos Vilavicencio (Chile).

Mário Brock, após realizar sua formação neurocirúrgica com Feliciano Pinto e J osé Ribe Portugal, dirigiu-se, em 1964, para a Alemanha, trabalhando nas Universidades de Colônia, Mogúcia e Hannover. Torna-se, em 1978, professor titular e chefe do Serviço de Neurocirurgia da Universidade de Berlim (Universitatsklinikum Steglitz).

Depois de José Ribe Portugal, outro pioneiro da neurocirurgia no Rio de Janeiro foi Paulo Niemeyer, também um autodidata. Em 1939 passa a trabalhar no Pronto Socorro, quando se dedica especialmen- te à neurocirurgia do trauma. Em 1942 assume o setor de neurocirurgia da Santa Casa. Em 1945 cria o Serviço de Neurocirurgia do Pronto Socorro, dedicado essencialmente ao neurotrauma.

Henri Gastaut (1915 - 1995), epileptologista francês, cujos trabalhos mostraram que a amígdala, o hipocampo e o giro parahipocampal são os substratos anatômicos das crises temporais, esteve no Brasil na década de 1950. Suas conferências sobre as bases anatomopatológicas da epilepsia temporal e eletrocorticografia inspiraram Niemeyer a idealizar a amigdalo-hipocampectomia transventricular para o tratamento destas crises $^{15}$ (Fig 7). Foi um dos fundadores da Sociedade Brasileira de Neurocirurgia.

\section{REFERÊNCIAS}

1. Ballance SC. A glimpse into the history of the surgery of the brain Lancet 1922;22:111-116;165-172.

2. Horrax G. Neurosurgery: an historical sketch. Springfield: Charles C. Thomas, 1952:10-15.

3. Greenblatt SH. A history of neurosurgery. Park Ridge: American Association of Neurological Surgeons, 1997:3-9.

4. Ribeiro L. Medicina no Brasil. Rio de Janeiro: Imprensa Nacional, 1940 53-59.

5. Salles P. História da medicina no Brasil. Belo Horizonte: Ed. G. Holman, 1971:160-161.

6. Gusmão SS, Souza JG. História da neurocirurgia no Brasil. Joinville: Letra d'Agua, 2000:120-128.

7. Brandão A Filho. Quisto da hipófise; ventriculografia e intervenção cirúrgica por via frontal. Jornal dos Clínicos 1924;16:224-251.

8. Egas Moniz. Confidências de um investigador científico. Lisboa: Edições Ática, 1949:129-136.

9. Brandão A Filho. Primeira encefalografia arterial no Brasil. In Brandão A Filho. Clínica cirúrgica. Rio de Janeiro: Editora Scientífica, 1930:271293.

10. Brandão A Filho. Tumores do encéfalo: algumas observações comentadas. Rio de Janeiro: Pimenta de Mello, 1931:1-77.

11. Brandão A Filho. Tique doloroso da face; secção da raiz sensitiva do trigêmeo. In Brandão A Filho. Clínica cirúrgica. Rio de Janeiro: Editora Scientífica, 1923:251-314.

12. Gomes MM. Marcos históricos da neurologia. Rio de Janeiro: Editora Científica Nacional, 1997:61-65

13. Reimão R. História da neurologia no Brasil. São Paulo: Lemos-Editorial, 1999:43-51.

14. Brock M. José Ribeiro Portugal: pai da neurocirurgia brasileira. Arq Neuropsiquiatr 1994;52:118-122.

15. Niemeyer $P$. The transventricular amygdala-hipocampectomy in temporal lobe epilepsy. In Baldwin MBP (ed.) Temporal lobe epilepsy. Springfield: Charles C Thomas, 1958:461-482. 